\title{
Apego a higiene de manos en 5 momentos y protección específica en un hospital de tercer nivel ante la pandemia de COVID-19
}

\author{
Javier A. Maciel-Urzúa, ${ }^{1}$ Gabriela Zamudio-Martínez, ${ }^{2}$ Goretti Rangel-León, ${ }^{3}$ \\ Mauricio E. Bustamante-Morales, ${ }^{1}$ Rodrigo A. Del Valle-Díaz de León ${ }^{4 *}$ y José V. Pérez-Navarro ${ }^{1}$ \\ ${ }^{1}$ Instituto Mexicano del Seguro Social, Centro Médico Nacional de Occidente, Servicio de Cirugía General, Jalisco; ${ }^{2}$ Instituto Mexicano del Seguro \\ Social, Hospital General Regional 46, Servicio de Cirugía General, Jalisco; ${ }^{3}$ Instituto de Seguridad y Servicios Sociales de los Trabajadores del \\ Estado, Hospital Regional de León, Servicio de Medicina Interna, Guanajuato; " Hospital General "Dr. Manuel Gea González", Torre de Especialidades, \\ División de Oftalmología, Ciudad de México. México
}

\section{Resumen}

Introducción: Ante la pandemia de COVID-19, el apego a las medidas de higiene es un objetivo para disminuir la morbimortalidad. Objetivo: Evaluar el apego a la higiene de manos y medidas de protección durante la pandemia de COVID-19 en un hospital de tercer nivel. Métodos: Estudio transversal acerca del lavado de manos del personal de salud en los cinco tiempos recomendados por la Organización Mundial de la Salud, así como sobre el uso del equipo de protección personal específico. Resultados: Fueron observadas 117 oportunidades de higiene de manos en personal de salud: 40 (34 \%) respecto al lavado de manos y 76 (65\%) respecto a su omisión; sobre el apego al uso de careta en cinco (4\%) y sobre la falta de apego en 112 (96\%). Se identificó apego al uso de mascarilla en 65 profesionales de enfermería (87\%), uso adecuado de mascarilla en 56 de ellos (60 \%) y uso de careta en uno (1\%). Conclusión: El personal mostró baja proporción de apego a la higiene de manos y al uso de equipo para la protección específica durante la pandemia de COVID-19.

PALABRAS CLAVE: Higiene de manos. Protección específica. COVID-19.

\section{Adherence to hand hygiene at five moments and specific protection in a tertiary care hospital during the COVID-19 pandemic}

\begin{abstract}
Introduction: During the COVID-19 pandemic, adherence to hygiene measures is an objective aimed at reducing morbidity and mortality. Objective: To evaluate adherence to hand hygiene and protection measures during the COVID-19 pandemic in a tertiary care hospital. Methods: Cross-sectional study on health personnel handwashing at the five moments recommended by the World Health Organization, as well as on the use of specific personal protective equipment. Results: One hundred and seventeen hand hygiene opportunities were observed in health personnel. Hand washing was observed in 40 (34\%) and omission in 76 (65\%). Adherence to the use of face shield was observed in five (4\%), and lack of adherence in $112(96 \%)$. Adherence to the use of face mask was observed in 65 nursing professionals (87\%), with appropriate use of the mask in 56 of them (60\%) and use of face shield in one (1\%). Conclusion: Health personnel showed low proportions of adherence to hand hygiene and use of equipment for specific protection during the COVID-19 pandemic.
\end{abstract}

KEY WORDS: Hand hygiene. Specific protection. COVID-19.

Correspondencia:

*Rodrigo A. Del Valle-Díaz de León

E-mail: r.alexxis91@gmail.com
Gac Med Mex. 2021;157:327-331

Disponible en PubMed

www.gacetamedicademexico.com

0016-3813/@ 2021 Academia Nacional de Medicina de México, A.C. Publicado por Permanyer. Este es un artículo open access bajo la licencia CC BY-NC-ND (http://creativecommons.org/licenses/by-nc-nd/4.0/). 


\section{Introducción}

Resulta importante que los trabajadores sanitarios conozcan las medidas de protección personal, su uso adecuado e indicaciones, cuya importancia quedó demostrada durante la pandemia de COVID-19 por el alto índice de contagio en el personal de salud, aun con el uso de medidas de protección. Se ha reportado que el comportamiento en cuanto al apego a medidas de higiene en trabajadores de la salud no cambia significativamente a pesar de campañas de información y prevención en salud pública. ${ }^{1}$

La Organización Mundial de la Salud recomienda diversas estrategias para el lavado de manos. La higiene de manos debe llevarse a cabo en cinco momentos: antes de tocar al paciente, antes de realizar una tarea aséptica, después del riesgo de exposición a líquidos corporales, después de tocar al paciente y después del contacto con el entorno del paciente. $^{2}$ Aun cuando estas estrategias son accesibles a los diferentes sistemas de salud, tanto en países del primer mundo como en los países en desarrollo, no se han promocionado según las metas establecidas. $^{3}$

A pesar de disponer de equipos sofisticados para protección personal en enfermedades infectocontagiosas, no hay estudios con suficiente calidad para evidenciar cuál es el mejor. ${ }^{4}$ Evaluar la importancia del cumplimiento de la higiene de manos y del empleo adecuado del equipo de protección es un tema controvertido, ya que el personal de salud ha presentado COVID-19 a pesar de estas medidas.

La demostración de la eficacia de las recomendaciones y estrategias para mejorar la higiene del lavado de manos y la protección específica es crucial, tanto para motivar el comportamiento de los trabajadores de salud como para asegurar las medidas preventivas. Sin embargo, la investigación en este campo representa una actividad desafiante.

Para prevenir estos contagios entre el personal de salud, el Centro para el Control y Prevención de Enfermedades de Atlanta, en Estados Unidos, recomienda el uso de mascarillas $\mathrm{N}-95$ o de un nivel de protección superior en salas con pacientes sospechosos o confirmados con COVID-19. ${ }^{5}$ La Organización Mundial de la Salud propone el uso de guantes, delantales, gafas de protección, batas descartables y mascarillas N-95 durante el manejo de estos pacientes. ${ }^{6}$

El lavado de manos y las precauciones específicas son herramientas importantes en la prevención de infecciones. Sin embargo, a pesar de la evidencia en la reducción de transmisión de las infecciones con estas medidas y de que son muy costo-efectivas, el apego del personal de salud es bajo. ${ }^{3,4.7}$ Se ha demostrado que hasta $50 \%$ de las infecciones pueden ser prevenibles; el lavado de manos se considera el mejor método de prevención. Por otro lado, diversos estudios señalan el riesgo aumentado en trabajadores de la salud de contraer y esparcir enfermedades infecciosas. . $^{810}$

En un inicio, en el Instituto Mexicano del Seguro Social no se disponía de una guía práctica sobre el empleo de equipo de protección del personal de salud para la atención de pacientes con COVID-19, por lo que la Secretaría de Salud propuso la preparación en ese sentido -con énfasis en medidas de prevención de infecciones, higiene de manos, aislamiento de contacto y pruebas de ajuste de mascarillas N95- del personal que realiza procedimientos generadores de aerosoles. ${ }^{11}$ Actualmente se puede acceder a la guía "Lineamiento para atención de pacientes COVID".11 Para la protección del personal de salud, la Organización Mundial de la Salud recomienda tener especial atención en la higiene de manos, empleo de mascarilla, bata sanitaria, guantes desechables y gafas de protección ocular. ${ }^{12}$ Las indicaciones de uso de estos implementos se deben basar en el entorno, los destinatarios, el riesgo de exposición (por ejemplo, el tipo de actividad) y la dinámica de transmisión del patógeno (por contacto, gotículas respiratorias, aerosoles, etcétera). ${ }^{13}$

El equipo de protección lo conforman los siguientes elementos:

- Lentes de protección general: con nivel mínimo de protección ocular recomendado para uso en laboratorio, por lo que tienen que incluir protección lateral. Se reconoce que no son completamente efectivos contra contacto por salpicadura. ${ }^{14}$

- Guantes: adaptados a diferentes situaciones clínicas y pueden ser de látex, vinil o nitrilo.

- Mascarilla quirúrgica: se usa para protección de la muestra biológica y del paciente (si bien no protege a quien la porta), contra la emisión de gotas respiratorias.

- Protección corporal: bata clínica, cuyo uso está indicado en todos los laboratorios, de acuerdo con el riesgo de exposición a material flamable o no; la más común es la tradicional de algodón 0 poliéster. ${ }^{2}$ 
El propósito de esta investigación es conocer el apego del personal de salud a las medidas de prevención como el lavado de manos y el uso adecuado del equipo de protección personal dentro de un hospital del Centro Médico Nacional de Occidente, entre noviembre de 2020 y enero de 2021.

\section{Métodos}

Se realizó un estudio transversal, observacional, descriptivo y prospectivo que incluyó a personal de enfermería, médicos, residentes y de otras categorías, que laboran en área de atención a pacientes de un hospital híbrido del Centro Médico Nacional de Occidente; mediante técnica de sombra se observó si se llevaba a cabo la higiene de las manos y la protección específica según la metodología establecida.

El muestreo se determinó por conveniencia y el tamaño de la muestra fue no probabilístico debido a que se evaluó el apego de todo el personal que laboraba en el hospital.

Se incluyeron médicos de todas las especialidades, de base y residentes, enfermeras de todos los rangos y personal de otro tipo y de todos los rangos, quienes laboraban en cualquier área hospitalarias. Se eliminó del estudio a los trabajadores que advirtieron la presencia del evaluador.

Las variables que se evaluaron siguieron los lineamientos de la herramienta de medición del Modelo Institucional para Prevenir y Reducir las Infecciones Nosocomiales, del Instituto Mexicano del Seguro Social, la cual su vez está basada en la recomendada por la Organización Mundial de la Salud y que especifica lo siguiente: higiene de manos (en cinco momentos), lavado de manos, frote de manos (alcohol en gel), mascarilla y gafas de protección.

Para evaluar este apego se asignaron dos investigadores principales, que forman parte del equipo de esta investigación y trabajan en el hospital donde se llevó a cabo el estudio, quienes fueron capacitados y recibieron material por escrito respecto a las indicaciones y los conceptos relacionados, para que fueran capaces de aplicar la herramienta de medición, así como para identificar y diferenciar los elementos por evaluar.

Cada observador fue situado cerca del punto de atención; se les recomendó que colocaran el formulario sobre un soporte sólido para facilitar la tarea de observación. Cada observador buscó alguna indicación y la convirtió en una oportunidad al tiempo que la registró, empleando un procedimiento especial de captura. Fue asignado un periodo fijo de obtención de datos, de acuerdo con el tamaño de la muestra, en busca de oportunidades para evaluar la acción realizada por el personal sanitario; a los observadores se les indicó que como máximo podían realizar el registro de tres trabajadores de la salud al mismo tiempo. El periodo de observación se definió como el espacio temporal durante el cual se midió el cumplimiento en un determinado escenario. Una vez concluida la observación en el sujeto estudiado, a este se le solicitó autorizar su participación como parte de la muestra con el otorgamiento del consentimiento informado verbal. No se recabaron nombres a fin de evitar sesgos en la medición.

Para el análisis de los resultados se utilizaron medidas de tendencia central (media, mediana, moda) y de dispersión (desviación estándar, valor mínimo y valor máximo), estimación de medias y proporciones, así como de frecuencias absolutas y relativas en las variables cualitativas. Se empleó el programa estadístico SPSS versión 24.0. El estudio fue aprobado por el comité de ética e investigación del hospital.

\section{Resultados}

Durante la observación se logró identificar 117 "oportunidades" de higiene de manos en 102 sujetos diferentes del personal de salud; de esas oportunidades, 61 (52 \%) fueron realizadas por personal del sexo femenino, 75 (64.1\%) por personal de enfermería y $34(29.1 \%)$ por médicos residentes; 45 (38.5\%) se llevaron a cabo por personal que laboraba en jornada y $75(64.1 \%)$ en área del piso. El resto de las características de las 117 observaciones se detalla en la Tabla 1.

La oportunidad de observación se dio antes de que el personal de salud tocara al paciente en $35 \%$ de las ocasiones, después de tocar al paciente en $40 \%$, después del contacto con el entorno del paciente en $22 \%$ y antes de una tarea aséptica en $3 \%$. Dentro de la observación de acción del personal de salud se registró lavado de manos en 40 ocasiones (34.18\%) y omisión en 76 ocasiones (64.9\%). Respecto al apego al uso de equipo de protección, se registró uso de careta en cinco observaciones (4\%) y sin uso de careta en 112 (96\%). En cuanto al apego al uso de mascarilla, en 65 (87\%) observaciones se trató de personal de enfermería; en 56 (60\%) el uso de mascarilla fue adecuado. Se registró uso de careta en una ocasión (1\%). 
Tabla 1. Características de 117 registros de higiene en personal de salud

\begin{tabular}{|l|c|c|}
\hline Característica & n & $\%$ \\
\hline Sexo & & \\
$\quad$ Masculino & 56 & 48.0 \\
Femenino & 61 & 52.0 \\
Categoría & & \\
$\quad$ Enfermero (a) & 75 & 64.1 \\
$\quad$ Residente & 34 & 29.1 \\
Otra & 6 & 5.1 \\
$\quad$ Médico adscrito & 2 & 1.7 \\
Turno en el que labora & & \\
$\quad$ Matutino & 33 & 28.2 \\
$\quad$ Vespertino & 16 & 13.7 \\
$\quad$ Nocturno & 23 & 19.7 \\
$\quad$ Jornada & 45 & 38.5 \\
Área en la que labora & & \\
$\quad$ Urgencias & & 24.8 \\
$\quad$ Piso & 29 & 64.1 \\
$\quad$ Unidad de cuidados COVID & 13 & 11.1 \\
\end{tabular}

\section{Discusión}

El apego al lavado de manos no debería tener una constante de cambio a través del tiempo, ya que se conocen sus beneficios desde siglos antes. Se debería realizar capacitación frecuente, para mantener constante el apego a las medidas de seguridad por parte del personal de salud, el paciente y la población en general. No obstante, el apego al lavado de manos es diferente en cuanto al lugar y el tiempo y se refuerza durante las epidemias o pandemias y después de ellas. En este estudio, se encontró una frecuencia general del lavado de manos en el personal de salud de $34.18 \%$. Un estudio realizado en 2007 en el Hospital de Especialidades del Centro Médico Nacional La Raza evaluó la prevalencia y el apego al lavado de manos mediante técnica de sombra, con una muestra de 299 trabajadores de la salud. La prevalencia general de lavado de manos fue de $60.2 \%$, en personal de enfermería de $62 \%$, en médicos residentes de $57.6 \%$, en médicos adscritos de $75 \%$ y en innaloterapeutas de $20 \%{ }^{15}$

En 2011, después de la epidemia de influenza H1N1 en 2009, se llevó a cabo un estudio multimodal de higiene de manos en el Hospital de Pediatría, Centro Médico Nacional Siglo XXI del Instituto Mexicano del Seguro Social: la prevalencia del lavado de manos fue de $51 \%$ y $38.9 \%$ lo hizo en los cinco momentos recomendados $(p<0.05){ }^{16}$
El apego al lavado de manos en este estudio fue superior al de reportes previos en esa misma institución, hallazgo similar al encontrado por Labarca et al., ${ }^{7}$ quienes encontraron un apego mayor durante la pandemia por influenza H1N1, en comparación a un periodo anterior y posterior a esta. Aunque no se encontró una causa de este efecto, se puede atribuir a las intensas campañas de educación en cuanto a prevención y al temor de contagiarse. Es importante considerar el efecto Hawthorne, una respuesta inducida por el conocimiento de los participantes de que están siendo estudiados.

\section{Conclusión}

Se observó mayor apego a las medidas de protección, superior al reportado en la misma unidad antes de la contingencia. En el personal de enfermería se registró la mayor frecuencia de apego; sin embargo, siguiendo esta línea de investigación, es importante señalar que se identificó que se requiere mayor conocimiento de los protocolos, lo que puede dar lugar al inicio de medidas de orientación y educación para la salud y reforzaría la protección del personal de salud ante la pandemia ocasionada por el virus SARS-CoV-2 y contra otras infecciones.

\section{Financiamiento}

La presente investigación no recibió ninguna beca específica de agencias de los sectores públicos, comercial o sin ánimo de lucro.

\section{Conflicto de intereses}

Los autores declaran no tener conflicto de intereses.

\section{Responsabilidades éticas}

Protección de personas y animales. Los autores declaran que para esta investigación no se han realizado experimentos en seres humanos ni en animales.

Confidencialidad de los datos. Los autores declaran que en este artículo no aparecen datos de pacientes.

Derecho a la privacidad y consentimiento informado. Los autores declaran que en este artículo no aparecen datos de pacientes. 


\section{Bibliografía}

1. Pires-Dos Santos R, Konkewicz LR, Nagel F, Lisboa T, Jacoby $T$, Ludwig-Gastal S, et al. The 2009 H1N1 influenza A pandemic and hand hygiene practices in a hospital in the South of Brazil. Infect Control Hosp Epidemiol. 2010:31:131301315.

2. Organización Mundial de la Salud [Internet]. Suiza: Prevención y control de infecciones en los centros de atención de larga estancia en el contexto de la COVID-19: orientaciones provisionales; 2020

3. Bolon MK. Hand hygiene: an update. Infect Dis Clin North Am. 2016;30:5910607.

4. Allegranzi B, Pittet D. Role of hand hygiene in healthcare-associated infection prevention. J Hosp Infect. 2009;73:305ロ315.

5. Heinzerling A, Stuckey P, Scheuer T, Xu K, Perkins K, Resseger H, et al. Transmission of COVID-19 to health care personnel during exposures to a hospitalized patient - Solano County, California, February 2020. MMWR Morb Mortal Wkly Rep. 2020;69:472.

6. World Health Organization [Internet]. Suiza: Rational use of personal protective equipment for coronavirus disease (COVID-19) and considerations during severe shortages; 2020.

7. Labarca J, Zambrano A, Niklitschek S, Ferrés M, Pérez C, Ajenjo MC et al. H1N1 pandemic influenza impact on hand hygiene and specific precautions compliance among healthcare workers. J Hosp Infect. 2011;79(2):1770179.

8. Lydon S, Power M, McSharry J, Byrne M, Madden C, O'Connor P, et al Interventions to improve hand hygiene compliance in the ICU: a systematic review. Crit Care Med. 2017;45:e1165ロe1172.
9. Jiang L, Ng IHL, Hou Y, Li D, Lin-Tan LW, An-Ho HJ, et al. Infectious disease transmission: survey of contacts between hospital-based healthcare workers and working adults from the general population. J Hosp Infect. 2018;98:404凸411.

10. Huttunen R, Syriänen J. Healthcare workers as vectors of infectious diseases. Eur J Clin Microbiol Infect Dis. 2014;33(9):147701488.

11. Lineamiento para la atención de pacientes por COVID 19 [Internet]. México: Comisión Coordinadora de Institutos Nacionales de Salud y Hospitales de Alta Especialidad/Secretaria de Salud; 2020.

12. Uso racional del equipo de protección personal frente a la COVID-19 y aspectos que considerar en situaciones de escasez graves: orientaciones provisionales, 6 de abril de 2020 [Internet]. Suiza: World Health Organization; 2020.

13. Organización Mundial de la Salud [Internet]. Suiza: Prevención y control de infecciones durante la atención sanitaria de casos en los que se sospecha una infección por el nuevo coronavirus (nCoV): orientaciones provisionales. [Disponible: 2020 Ene 25].

14. Allegranzi B, Gayet-Ageron A, Damani N, Bengaly L, McLaws ML, Urroz O, et al. Global implementation of WHO's multimodal strategy for improvement of hand hygiene: a quasi-experimental study. Lancet Infect Dis. 2013;13:843ロ851.

15. Anaya-Flores VE, Ortiz-López S, García-Hernández A, Jiménez-Bravo ML, Ángeles-Garay $\mathrm{U}$, et al. Prevalencia de lavado de manos y factores asociados con el incumplimiento. Estudio de sombra. Rev Enferm Inst Mex Seguro Soc. 2007;15:141-146.

16. Zamudio-Lugo I, Meza-Chávez A, Martínez-Sánchez Y, Miranda-Novales MG, Espinosa-Vital JG, Rodríguez-Sing R. Estudio multimodal de higiene de manos en un hospital pediátrico de tercer nivel. Bol Med Hosp Infant Mex. 2012;69:384-390. 